$18^{\text {th }}$ European Symposium on Computer Aided Process Engineering - ESCAPE 18

Bertrand Braunschweig and Xavier Joulia (Editors)

(C) 2008 Elsevier B.V./Ltd. All rights reserved.

\title{
Analysis of the Constraint Characteristics of a Sheet Forming Control Problem Using Interval Operability Concepts
}

\author{
Fernando V. Lima ${ }^{\mathrm{a}}$, Christos Georgakis ${ }^{\mathrm{a}}$, Julie F. Smith ${ }^{\mathrm{b}}$, Phillip D. Schnelle ${ }^{\mathrm{b}}$ \\ ${ }^{a}$ Systems Research Institute \& Department of Chemical and Biological Engineering, \\ Tufts University, Medford, MA 02155, USA \\ ${ }^{b}$ DuPont Engineering Research and Technology, Brandywine 7304, 1007 Market Street, \\ Wilmington, DE 19898, USA
}

\begin{abstract}
An Interval Operability-based approach $[1,2]$ is applied to calculate operable output constraints for the Sheet Forming Control Problem (SFCP) from DuPont. The SFCP attempts to control the sheet thickness at 15 different points, which represent 15 output variables, using 9 manipulated variables in the presence of 3 disturbances. Thus, this problem represents a computationally complex, high-dimensional non-square system with more outputs than inputs. The SFCP is addressed here under two study cases: 1) a non-square, where all the 15 outputs are controlled independently of each other; 2) a square, where 6 outputs are combined in pairs, or zone variables, and controlled within their corresponding zone. Results show that significant reduction of the constrained region of process operation can be achieved for different output targets specified. Specifically, the hyper-volume ratio of the initial to the designed constrained regions range between $10^{3}-10^{5}$. The calculated constraints are validated by running DMCplus $^{\mathrm{TM}}$ (AspenTech) simulations for the extreme values of the disturbances. These constraints are intended for use online in model-based controllers (e.g., Model Predictive Control) to calculate the tightness with which each of the outputs can be controlled.
\end{abstract}

Keywords: Operability, Sheet Forming Process, Non-square Systems, Model Predictive Control.

\section{Introduction}

In recent years, chemical process designs have increased in complexity due to material and energy conservation requirements, integration of units, process optimization and stricter environmental regulations. Consequently, tools to systematically assess the capabilities of such designs and its integration with the process control structure have become increasingly important. These tools should identify a design's ability to achieve the feasible region of operation around a steady-state in the presence of process disturbances. Specifically, it is important to determine the tightest feasible set of output constraints that can be achieved considering the constraint limitations of the input variables, which are design dependent [2]. The improper selection of these output constraints can make the controller infeasible when a disturbance moves the process outside its usual operating region. Hard constraints are enforced whenever feasible and softened whenever it is necessary to retain feasibility [3]. The Operability methodology originally introduced for square systems (Set-Point Operability [4]) and extended for non-square systems (Interval Operability $[1,2,5,6]$ ) enables the systematic selection of 
such output constraints, so that they are as tight as possible and do not render the controller infeasible.

Using the previously published Interval Operability concepts and algorithms, this paper aims to study the constraint characteristics of a Sheet Forming process from DuPont, which is characterized by a high-dimensional and non-square system. For such system, set-point control is not possible for all the outputs and interval control is necessary. This is done by analyzing two configurations of this problem, a $15 \times 9 \times 3$ (outputs $x$ inputs $x$ disturbances) non-square system and a simplified $9 \times 9 \times 3$ square sub-system. This simplified system is obtained by exploring the distributed characteristics of the SFCP by considering 6 zone variables. The presence of disturbances of high magnitude may preclude set-point control even for square systems. For such cases, the concept of Interval Operability may be equally applicable to calculate the tightest feasible output ranges.

\section{Interval Operability Concepts}

The necessary sets to enable the Interval Operability assessment for an $n \times m \times q$ (outputs $x$ inputs $x$ disturbances) system are defined in this section [5]. The Available Input Set (AIS) is the set of values that the process input variables can take based on the constraints of the process. The Desired Output Set (DOS) is given by the ranges of the outputs that are desired to be achieved and the Expected Disturbance Set (EDS) represents the expected steady-state values of the disturbances. These sets are mathematically represented by:

$$
\begin{aligned}
& \text { AIS }=\left\{\mathbf{u} \mid u_{i}^{\min } \leq u_{i} \leq u_{i}^{\max } ; 1 \leq i \leq m\right\} ; \operatorname{DOS}=\left\{\mathbf{y} \mid y_{i}^{\min } \leq y_{i} \leq y_{i}^{\max } ; 1 \leq i \leq n\right\} ; \\
& \mathbf{E D S}=\left\{\mathbf{d} \mid d_{i}^{\min } \leq d_{i} \leq d_{i}^{\max } ; 1 \leq i \leq q\right\}
\end{aligned}
$$

The Achievable Output Set for a specific disturbance value, $\operatorname{AOS}(\mathbf{d})$, is defined by the ranges of the outputs that can be achieved using the inputs inside the AIS:

$$
\operatorname{AOS}(\mathbf{d})=\left\{\mathbf{y} \mid \mathbf{y}=\mathbf{G u}+\mathbf{G}_{d} \mathbf{d} ; \mathbf{u} \in \operatorname{AIS} \text {, fixed } \mathbf{d} \in \operatorname{EDS}\right\}
$$

where the matrices $\mathbf{G}$ and $\mathbf{G}_{d}$ represent the linear steady-state process and disturbance gain matrices, respectively. Finally, the Achievable Output Interval Set (AOIS) is defined as the tightest possible feasible set of output constraints that can be achieved, with the available range of the manipulated variables and when the disturbances remain within their expected values. The algorithm developed for the calculation of this important set is presented next.

\section{Calculation of AIS: Linear Programming Approach}

Two sets of output parameters are considered in the AOIS calculation: the steady-state target point $\left(\mathbf{y}_{0}\right)$ and the relative output weights $(\mathbf{w})$. The relative output weights represent the relative tightness with which each output will be controlled around its desired target and will affect the aspect ratio of the corresponding sides of the designed AOIS. For example, an aspect ratio of 1:10 between two outputs assures that one will be controlled 10 times more tightly, approximating set-point control. Several examples of AOIS calculations using different weights and output targets have been previously published $[1,2]$. The set of points that characterize the vertices of the AOS can be easily calculated by directly mapping the vertices of the AIS and EDS using the linear steady-state process model (eq. 1). The calculation of $\mathrm{AOIS}$ in $\mathfrak{R}^{n}$ is performed by 
formulating the interval operability problem in a Linear Programming (LP) framework, where the AOS and the AOIS polytopes are described as a system of inequalities in the LP formulation. An overview of the algorithm for this calculation, presented in reference [6], is the following:

1) Define the relative weights $w_{1}, w_{2}, \ldots w_{n}$ that quantify the relative tightness within which each output needs to be controlled;

2) Select one of the extreme disturbance vectors $\mathbf{d}=\mathbf{d}_{i}, i=1,2, \ldots, k$, which corresponds to one of the $k=2^{\mathrm{q}}$ vertices of EDS. Calculate $\operatorname{AOS}\left(\mathbf{d}_{i}\right)$ (eq. 1) and the corresponding linear equalities and inequalities that define this set (see details in reference [6]);

3) Define a family of $n$-dimensional orthogonal parallelepipeds, $P(\alpha)$, self-similar among them, centered at the target value of the outputs $\left(\mathbf{y}_{0}\right)$, where the scalar $\alpha$ affects each of their sizes by the following set of inequalities:

$P(\alpha)=\left\{\mathbf{y} \mid-\mathbf{b} \leq \mathbf{y}-\mathbf{y}_{\mathbf{0}} \leq \mathbf{b}\right\}$

where

$\mathbf{b}=\left(\frac{\alpha}{w_{1}}, \frac{\alpha}{w_{2}}, \cdots, \frac{\alpha}{w_{n}}\right)^{T} ; \mathbf{y}_{\mathbf{0}}=\left(y_{01}, y_{02}, \cdots, y_{0 n}\right)^{T} ;$ and $\mathbf{y}=\left(y_{1}, y_{2}, \cdots, y_{n}\right)^{T}$

4) Calculate the minimum value of $\alpha, \alpha_{i}$, such that $P\left(\alpha_{i}\right)$ and $\operatorname{AOS}\left(\mathbf{d}_{i}\right)$ have a single common point $\mathbf{v}_{i}$, by solving the LP problem below:

$$
\begin{aligned}
& \alpha_{i}=\min _{\mathbf{x}} \alpha=\min _{\mathbf{x}} \mathbf{f}^{\mathbf{T}} \mathbf{x},
\end{aligned}
$$

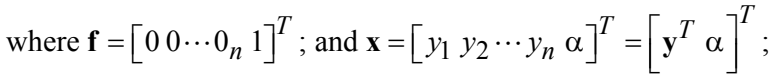

$$
\begin{aligned}
& \text { while } \mathbf{y}=\mathbf{v}_{i} \in P\left(\alpha_{i}\right) \cap \operatorname{AOS}\left(\mathbf{d}_{i}\right)
\end{aligned}
$$

5) Repeat steps 2 to 4 above for a total of $k=2^{q}$ times to calculate the set of $k$ points: $\mathbf{v}_{1}, \mathbf{v}_{2}, \ldots \mathbf{v}_{k}$;

6) The final AOIS is the smallest orthogonal parallelepiped in $\Re^{n}$ that includes all the $k \mathbf{v}_{i}$ points from the LP solutions (AOIS $=O P\left(\mathbf{v}_{1}, \mathbf{v}_{2}, \mathbf{v}_{3}, \ldots \mathbf{v}_{k}\right)$ ). This set defines the tightest set of output constraints that makes the process operable for the output target $\mathbf{y}_{0}$ and all the disturbance values inside the EDS.

\section{Results: Sheet Forming Control Problem}

As briefly described above, the objective of the Sheet Forming Control Problem (SFCP) is to control the sheet thickness at 15 different points as uniformly as possible around different targets $\left(\mathbf{y}_{0}\right)$. Thus, there are 15 controlled variables (CV's), which correspond to the thicknesses in the cross-direction, with the same relative weight (w). Moreover, this process has 9 manipulated variables (MV's) and 3 disturbance variables (DV's). The steady-state gain model, the sets within which the input and output variables are constrained and the relative weights of the output variables are given in the following equations: 


$$
\begin{aligned}
& \left(\begin{array}{l}
\delta y_{1} \\
\delta y_{2} \\
\delta y_{3} \\
\delta y_{4} \\
\delta y_{5} \\
\delta y_{6} \\
\delta y_{7} \\
\delta y_{8} \\
\delta y_{9} \\
\delta y_{10} \\
\delta y_{11} \\
\delta y_{12} \\
\delta y_{13} \\
\delta y_{14} \\
\delta y_{15}
\end{array}\right)\left(\begin{array}{ccccccccc}
-2 & 0 & -1.8 & 0 & 0 & 0 & -0.00064 & 0 & 0 \\
-2 & 0 & -1.6 & 0 & 0 & 0 & -0.00035 & 0 & 0 \\
-2 & 0 & -1.4 & -2 & 0 & 0 & 0 & 0 & 0 \\
-2 & 0 & 0 & -1.8 & 0 & 0 & 0 & -0.00036 & 0 \\
-2 & 0 & 0 & -1.6 & 0 & 0 & 0 & -0.00064 & 0 \\
-2 & 0 & 0 & 0 & -1.6 & 0 & 0 & -0.00084 & 0 \\
0 & -2 & 0 & 0 & -1.8 & 0 & 0 & -0.00096 & 0 \\
0 & -2 & 0 & 0 & -2 & 0 & 0 & -0.001 & 0 \\
0 & -2 & 0 & 0 & -1.8 & 0 & 0 & -0.00096 & 0 \\
0 & -2 & 0 & 0 & 0 & 0 & 0 & -0.00084 & 0 \\
0 & -2 & 0 & 0 & 0 & -1.8 & 0 & -0.00064 & 0 \\
0 & -2 & 0 & 0 & 0 & -1.8 & 0 & -0.00036 & 0 \\
0 & -2 & 0 & 0 & 0 & -2 & 0 & 0 & 0 \\
0 & -2 & 0 & 0 & 0 & -1.8 & 0 & 0 & -0.00036 \\
0 & -2 & 0 & 0 & 0 & 0 & 0 & 0 & -0.00064
\end{array}\right)\left(\begin{array}{l}
\delta u_{1} \\
\delta u_{2} \\
\delta u_{3} \\
\delta u_{4} \\
\delta u_{5} \\
\delta u_{6} \\
\delta u_{7} \\
\delta u_{8} \\
\delta u_{9}
\end{array}\right)+\left(\begin{array}{ccc}
1.7 & 0 & 0 \\
1.7 & 0 & 0 \\
1.7 & 0 & 0 \\
1.7 & 0 & 0 \\
1.7 & 0 & 0 \\
0 & 1.7 & 0 \\
0 & 1.7 & 0 \\
0 & 1.7 & 0 \\
0 & 1.7 & 0 \\
0 & 1.7 & 0 \\
0 & 0 & 1.7 \\
0 & 0 & 1.7 \\
0 & 0 & 1.7 \\
0 & 0 & 1.7 \\
0 & 0 & 1.7
\end{array}\right)\left(\begin{array}{l}
\delta d_{1} \\
\delta d_{2} \\
\delta d_{3}
\end{array}\right) \\
& \mathrm{AIS}=\left\{\begin{array}{c}
u \in \mathfrak{R}^{9} \mid 210 \leq u_{1} \leq 230 ; 210 \leq u_{2} \leq 230 ; 220 \leq u_{3} \leq 235 ; \\
220 \leq u_{4} \leq 235 ; 220 \leq u_{5} \leq 235 ; 220 \leq u_{6} \leq 235 ; \\
2000 \leq u_{7} \leq 4000 ; 2000 \leq u_{8} \leq 4000 ; 2000 \leq u_{9} \leq 4000
\end{array}\right\} \\
& \operatorname{EDS}=\left\{\mathbf{d} \in \mathfrak{R}^{3} \mid-\beta \leq d_{i} \leq \beta \text {; for } 1 \leq i \leq 3 \text {; and } 0 \leq \beta \leq 12\right\} ; \mathbf{d}_{s s}=(0,0,0)^{T} \\
& \operatorname{DOS}=\left\{\mathbf{y} \in \mathfrak{R}^{15} \mid 1.9 \leq y_{i} \leq 2.3 \text {; for } 1 \leq i \leq 15\right\} \\
& \mathbf{w}=(1,1,1,1,1,1,1,1,1,1,1,1,1,1,1)^{T} \\
& \mathbf{y}_{s s}=(2.1,2.1,2.1,2.1,2.1,2.1,2.1,2.1,2.1,2.1,2.1,2.1,2.1,2.1,2.1)^{T} \\
& \mathbf{u}_{s s}=(220,220,227.5,227.5,227.5,227.5,3000,3000,3000)^{T} \\
& \mathbf{y}_{0}=(2.1,2.1,2.1,2.1,2.1,2.1,2.1,2.1,2.1,2.1,2.1,2.1,2.1,2.1,2.1)^{T}
\end{aligned}
$$

where $\delta \mathbf{y}, \delta \mathbf{u}$ and $\delta \mathbf{d}$ are deviation variables from the steady-state values for the outputs $\left(\mathbf{y}_{s s}\right)$, inputs $\left(\mathbf{u}_{s s}\right)$, and disturbances $\left(\mathbf{d}_{s s}\right)$, respectively. The scalar $\beta$ represents the magnitude of the disturbances, all three of which move in tandem. The design of the feasible set of output constraints will be performed here by considering the system above with its original dimensionality (section 4.1) and a $9 \times 9 \times 3$ square approximation (4.2).

4.1. System with its Original Dimensionality

To demonstrate the effectiveness of the proposed LP-based approach to handle highdimensional non-square systems, the SFCP (eq. 4) is addressed in its full dimensionality $(15 \times 9 \times 3)$. The calculated minimum $\left(\mathbf{y}^{\min }\right)$ and maximum $\left(\mathbf{y}^{\max }\right)$ AOIS ranges for each controlled variable when $\beta=12$ are shown in the first two rows of Table 1 . Because sheet-thickness uniformity is desirable, the following conservative set of output constraints, representing the widest thicknesses $\left(y_{10}, y_{11}, y_{13}\right)$, should be used:

$$
\text { AOIS }=\left\{\mathbf{y} \in \mathfrak{R}^{15} \mid 2.00 \leq y_{i} \leq 2.20 ; 1 \leq i \leq 15\right\}
$$


Thus, the hyper-volume ratio (HVR) [5] of the original (DOS) to the designed (AOIS) constrained regions is: $H V R=(0.4 / 0.2)^{15}=3.28 \times 10^{4}$. Hence, for the assumed disturbance values, this process could be feasibly operated within a constrained region that is significantly tighter than the one initially specified. These designed new limits were validated by running DMCplus ${ }^{\mathrm{TM}}$ (AspenTech) simulations for the extreme values of the disturbances, showing that the MPC controller does not violate these limits at the steady-state. Furthermore, the computational time for the AOIS calculation was only 0.18 seconds (Dell PC with a 3.0-GHz Intel Pentium 4 processor). If tighter control of the overall sheet thickness is intended, process modifications should be performed to enlarge the AIS, and thus shrink the AIS, availing tighter control of $y_{10}, y_{11}$, and $y_{13}$, at least.

\subsection{Square Approximation}

Consider now a $9 \times 9 \times 3$ square approximation of the SFCP, where the objective is to control each of the 9 outputs at ranges within the DOS using 9 inputs. In this case, it is assumed that the sheet thicknesses are controlled at specified zones, which are represented by the following set of zone variables $(\mathbf{z})$ :

$$
\begin{aligned}
& z_{1}=\frac{y_{1}+y_{2}}{2} ; z_{2}=y_{3} ; z_{3}=\frac{y_{4}+y_{5}}{2} ; z_{4}=\frac{y_{6}+y_{7}}{2} ; z_{5}=y_{8} ; \\
& z_{6}=\frac{y_{9}+y_{10}}{2} ; z_{7}=\frac{y_{11}+y_{12}}{2} ; z_{8}=y_{13} ; z_{9}=\frac{y_{14}+y_{15}}{2} ;
\end{aligned}
$$

where $y_{8}\left(z_{5}\right)$ corresponds to the measurement at the center of the sheet. The zone variables have the same DOS limits, relative weights and the target values of the initial output variables. The solution of this approximated problem also provides an alternative way to calculate the achievable constraints for the output variables using the properties of this distributed process.

The AOIS calculation is performed setting again $\beta=12$, which corresponds to its maximum value. The following $A$ OIS ranges are obtained for this case:

$$
\text { AOIS }=\left\{\mathbf{z} \in \mathfrak{R}^{9} \mid 2.04 \leq z_{i} \leq 2.16 ; 1 \leq i \leq 9, \beta=12\right\}
$$

Here as well, the most conservative set of constraints is used, to guarantee feasibility and sheet uniformity, which corresponds to the limits of $z_{5}, z_{6}, z_{7}$ and $z_{8}$ (see Table 1). Observe that once again very tight control can be achieved, which is demonstrated by the high value of the ratio between the hyper-volumes of the original constrained region and the designed constrained region $\left(H V R=5.08 \times 10^{4}\right)$. This implies that for the assumed disturbance values, the process could be operated feasibly within a constrained region $5.08 \times 10^{4}$ tighter than the region initially specified by the DOS.

Because different targets for the sheet thickness are intended, the zone target will now be moved from its nominal value of 2.1 units to 2.0 units. For this target, the AOIS ranges for the zone variables obtained when the disturbance range is equal to $-12 \leq d_{1} \leq$ $12(\beta=12)$ are the following:

$$
\text { AOIS }=\left\{\mathbf{z} \in \mathfrak{R}^{9} \mid 1.92 \leq z_{i} \leq 2.08 ; 1 \leq i \leq 9, \beta=12\right\}
$$

Thus, for the specified conditions, the original constrained region could be again significantly reduced $\left(H V R=3.81 \times 10^{3}\right)$. As in the previous case, the results for this target of 2.0 units correspond to the conservative set of constraints, representing the widest calculated thicknesses among the zones. 
To conclude the analysis for this process example, the typical computational time for the AIS calculations of all cases was 0.19 seconds. Here as well, simulations were performed using DMCplus to validate the results for all cases. Finally, observe that the simplification of a non-square system by a square one provides a less realistic calculation of the AOIS (eqs. 5 and 7). In fact, if the results obtained for the zone variables were used for the outputs within the corresponding zone, then infeasibilities would occur for some outputs.

Table 1. Feasible output ranges (AOIS) calculated for the SFCP $(\beta=12)$. The output variables for the original problem with 15 outputs, 9 inputs and 3 disturbance variables, are represented with $\mathbf{y}$. The zone variables for the simplified square problem are represented with $\mathbf{z}$.

\begin{tabular}{cccccccccccc}
\hline $\mathbf{y}_{\mathbf{i}} / \mathbf{z}_{\mathbf{i}}$ & $\mathbf{i}=\mathbf{1}$ & $\mathbf{2 , 3}$ & $\mathbf{4}$ & $\mathbf{5 , 7}$ & $\mathbf{6}$ & $\mathbf{8}$ & $\mathbf{9}$ & $\mathbf{1 0 , 1 1 , 1 3}$ & $\mathbf{1 2}$ & $\mathbf{1 4}$ & $\mathbf{1 5}$ \\
\hline $\mathbf{y}^{\min }$ & 2.05 & 2.03 & 2.04 & 2.08 & 2.04 & 2.06 & 2.08 & 2.00 & 2.10 & 2.05 & 2.01 \\
$\mathbf{y}^{\max }$ & 2.15 & 2.17 & 2.16 & 2.12 & 2.16 & 2.14 & 2.12 & 2.20 & 2.10 & 2.15 & 2.19 \\
$\mathbf{z}^{\min }$ & 2.09 & 2.09 & 2.10 & 2.04 & 2.04 & 2.04 & 2.09 & & & & \\
$\mathbf{z}^{\max }$ & 2.11 & 2.11 & 2.10 & 2.16 & 2.16 & 2.16 & 2.11 & & & & \\
\hline
\end{tabular}

\section{Conclusions}

Interval Operability concepts were applied to calculate the tightest feasible set of output constraints for the Sheet Forming Control Problem (SFCP). By considering two different configurations of this problem, square and non-square, different sets of constraints were obtained. Results for both scenarios showed that significant constraint reduction can be achieved for the initial set of output constraints without rendering the control problem infeasible in the presence of process disturbances. Although the use of zone variables reduces the complexity of the problem, the results obtained using this configuration are less accurate than the ones calculated by addressing the problem in its full dimensionality. The square nature of the simplified problem provides tighter feasible ranges for the zone variables than the constraints calculated for the individual output variables when the non-square problem is solved. If the calculated ranges of the zone variables were to be used to define the ranges of all 15 measured outputs, in a $15 \mathrm{x}$ 9 MPC (or DMC) controller, infeasibilities would occur. The minimal computational time required for the corresponding calculations enables the online adaptation of the controller constraints depending on the current state of the process.

\section{Acknowledgments}

The authors gratefully acknowledge the financial support from PRF-ACS through grant \# 45400-AC9. We also wish to acknowledge William M. Canney from AspenTech for providing the DMCplus software.

\section{References}

[1] F. V. Lima and C. Georgakis, In Proceedings of the 2006 IFAC International Symposium on Advanced Control of Chemical Processes (ADCHEM) (2006) 989-994.

[2] F. V. Lima and C. Georgakis, In Proceedings of the 2007 IFAC International Symposium on Dynamics and Control of Process Systems (DYCOPS) 3 (2007) 49-54.

[3] J. B. Rawlings, IEEE Control Syst. Mag., 20 (2000) 38-52.

[4] D. R. Vinson and C. Georgakis, J. Process Contr., 10 (2000) 185-194.

[5] F. V. Lima and C. Georgakis, J. Process Contr., doi:10.1016/j.jprocont.2007.09.004 (2007).

[6] F. V. Lima and C. Georgakis, Input-Output Operability of Control Systems, Automatica, submitted for publication (2007). 